Computer Science and Mathematics Division

Mathematical Sciences Section

\title{
PACKED STORAGE EXTENSION FOR SCALAPACK
}

\author{
Ed F. D'Azevedo \\ Jack J. Dongarra \\ Oak Ridge National Laboratory \\ P.O. Box 2008, Bldg. 6012 \\ Oak Ridge, TN 37831-6367
}

Date Published: January 1997

This work was supported in part by the National Science Foundation Grant No. ASC-9005933; by the Defense Advanced Research Projects Agency under contract DAAH04-95-1-0077, administered by the Army Research Office; by the Office of Scientific Computing, U.S. Department of Energy, under Contract DEAC05-84OR21400; and by the National Science Foundation Science and Technology Center Cooperative Agreement No. CCR-8809615, and Center for Computational Sciences at Oak Ridge National Laboratory for the use of the computing facilities.

\author{
Prepared by the \\ Oak Ridge National Laboratory \\ Oak Ridge, Tennessee 37831 \\ managed by \\ Lockheed Martin Energy Research Corp. \\ for the \\ U.S. DEPARTMENT OF ENERGY \\ under Contract No. DE-AC05-96OR22464
}




\section{Contents}

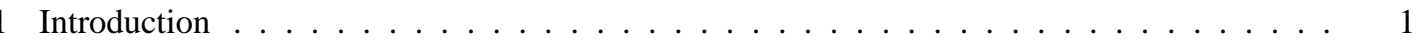

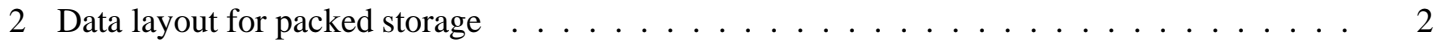

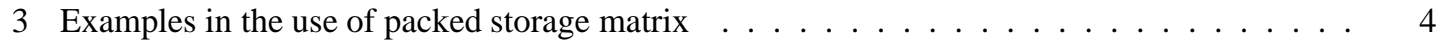

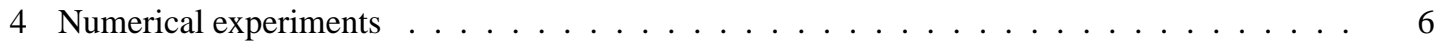

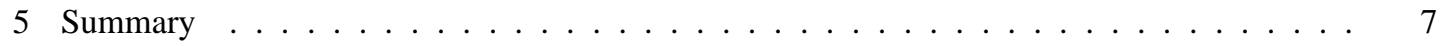

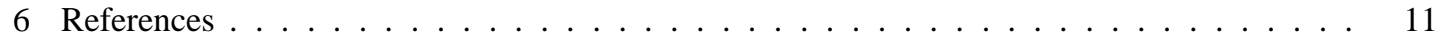




\section{List of Tables}

Performance (in seconds) of Cholesky factorizations and solves. . . . . . . . . . 8

Performance (in seconds) of simple drivers for symmetric eigensolvers. . . . . . . . 8

Performance (in seconds) of expert drivers for symmetric eigensolvers. . . . . . . . . . 8

Performance (in seconds) of expert drivers for generalized eigensolvers. . . . . . . . . 9 


\section{List of Figures}

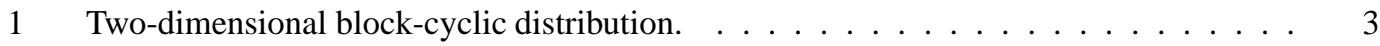

2 Example code to illustrate the reuse of ScaLAPACK components for matrices stored in packed storage. . . . . . . . . . . . . . . . . . 


\title{
PACKED STORAGE EXTENSION FOR SCALAPACK
}

\author{
Ed F. D’Azevedo
}

Jack J. Dongarra

\begin{abstract}
We describe a new extension to ScaLAPACK [2] for computing with symmetric (Hermitian) matrices stored in a packed form. The new code is built upon the ScaLAPACK routines for full dense storage for a high degree of software reuse. The original ScaLAPACK stores a symmetric matrix as a full matrix but accesses only the lower or upper triangular part. The new code enables more efficient use of memory by storing only the lower or upper triangular part of a symmetric (Hermitian) matrix. The packed storage scheme distributes the matrix by block column panels. Within each panel, the matrix is stored as a regular ScaLAPACK matrix. This storage arrangement simplifies the subroutine interface and code reuse. Routines PXPPTRF / PxPPTRS implement the Cholesky factorization and solution for symmetric (Hermitian) linear systems in packed storage. Routines PxSPEV/PxSPEVX (PxHPEV/PXHPEVX) implement the computation of eigenvalues and eigenvectors for symmetric (Hermitian) matrices in packed storage. Routines PxSPGVX (PxHPGVX) implement the expert driver for the generalized eigenvalue problem for symmetric (Hermitian) matrices in packed storage. Performance results on the Intel Paragon suggest that the packed storage scheme incurs only a small time overhead over the full storage scheme.
\end{abstract}




\section{Introduction}

This paper describes a new extension to ScaLAPACK [2] for computing with symmetric (Hermitian) matrices stored in a packed form. ScaLAPACK is an acronym for Scalable Linear Algebra PACKage, or Scalable LAPACK. ScaLAPACK is a library of high-performance linear algebra routines for distributed-memory message-passing MIMD (Multiple Instruction Multiple Data) computers and networks of workstations. Capability of ScaLAPACK is described in the ScaLAPACK Users' Guide [2].

The new code is built upon the ScaLAPACK routines for full dense storage for maximum portability. The original ScaLAPACK stores a symmetric matrix as a full matrix but accesses only the lower or upper triangular part. This design allows the reuse of Level 3 PBLAS (Parallel Basic Linear Algebra Subroutines) [3] without modification. However, almost half of the storage is holding redundant information. The new code enables more efficient use of memory by storing the submatrix blocks associated with only the lower or upper triangular part of a symmetric (Hermitian) matrix.

Although current computers have unprecedented storage and computation speed, they are also called upon to tackle ever larger problems. Let $N \times N$ be the largest symmetric (Hermitian) problem that can be stored in memory, then a larger approximately $\sqrt{2} N \times \sqrt{2} N$ symmetric matrix can be stored in the same memory using the packed storage scheme. Linear solution of symmetric (Hermitian) matrices by Cholesky factorization and computing eigenvalues and eigenvectors by the QR algorithm both have $O\left(N^{3}\right)$ complexities. With an $O\left(N^{3}\right)$ complexity, the runtime for solving the larger problem will be approximately $\sqrt{2}^{3} \approx 2.8$ times longer.

A symmetric eigensolver for packed storage may be adapted for use with out-of-core algorithms for solving large eigenvalue problems. The initial stage in the classical algorithm for finding eigenvalues and eigenvectors is to first reduce the original symmetric matrix into a tridiagonal matrix by orthogonal similarity Householder transformations. The original matrix is overwritten by these Householder transformations. One of the key steps is the frequent need for computing a matrix-vector multiply. An out-of-core algorithm that stores the symmetric matrix on disk would be highly inefficient since the matrix must be read in from disk for each matrix-vector multiply operation. A solution suggested by Ken Stanley is to hold in memory the symmetric matrix in packed storage and store the eigenvectors on disk. This approach would require $O\left(N^{2} / 2\right)$ memory for the symmetric matrix in packed storage instead of $O\left(2 N^{2}\right)$ memory for holding the symmetric matrix and eigenvectors in full storage, and would allow larger problems to be solved using the same limited amount of memory.

We have developed prototype codes PxPPTRF / PxPPTRS for Cholesky factorization and solution, and simple driver routines PxSPEV (PxHPEV) for finding eigenvalues and optionally eigenvectors of symmetric (Hermitian) matrices in packed storage. Expert drivers for symmetric (Hermitian) matrices PXSPEVX (PxHPEVX) and generalized eigenvalue problems PxSPGVX (PxHPGVX) are also available as prototype code. The names for the new routines follow the convention used in LAPACK [1] of using a 'P' to represent packed storage. Thus ' $\mathrm{SY}$ ' ('HE') represents a symmetric (Hermitian) matrix and 
'SP' ('HP') represents a symmetric (Hermitian) matrix in packed storage; similarly, 'PO' denotes a symmetric positive definite matrix and 'PP' denotes the symmetric positive definite matrix in packed storage.

Section 2 describes the layout of the packed storage scheme. Section 3 shows by a simple example how other ScaLAPACK routines can be modified for use with packed matrices. Section 4 summarizes the performance of PDPPTRF/PDPPTRS, PDSPEV, PDSPEVX and PDSPGVX on the Intel Paragon. Finally, Section 5 contains the summary.

\section{Data layout for packed storage}

ScaLAPACK principally uses a two-dimensional block-cyclic data distribution (see Figure 1) for full dense in-core matrices [2, Chapter 4]. This distribution has the desirable properties of good load balancing where the computation is spread reasonably evenly among the processes, and can make use of highly efficient level 3 BLAS (Basic Linear Algebra Subroutines) at the process level. Each colored rectangle represents an $m b \times n b$ submatrix. Matrix entry $(i, j)$ is mapped to matrix block $(i b, j b)=(1+$ $\lfloor(i-1) / m b\rfloor, 1+\lfloor(j-1) / n b\rfloor)$ and is assigned to process $(p, q)=\left(\bmod \left(i b-1, P_{r}\right), \bmod \left(j b-1, P_{c}\right)\right)$ on a $P_{r} \times P_{c}$ process grid. Thus the first entry $(1,1)$ is mapped to process $(0,0)$ and entry $(1+m b, 1+n b)$ is mapped to process $(1,1)$.

The packed storage scheme resembles the ScaLAPACK two-dimensional block-cyclic data distribution but physically stores only the lower (or upper) blocks. For example, on a $2 \times 3$ process grid as shown in Figure 1, if only the lower blocks are stored, then process $(0,0)$ holds blocks $A_{11}, A_{31}$, $A_{51}, A_{71}, A_{54}, A_{74}, A_{77}$. Process $(0,2)$ holds blocks $A_{33}, A_{53}, A_{73}$ and $A_{76}$. Similarly process $(1,1)$ holds blocks $A_{22}, A_{42}, A_{62}, A_{82}$ and $A_{65}, A_{85}$ plus $A_{88}$. We note that each block in the packed storage scheme is assigned to the same process as in the fully two-dimensional block-cyclic data distribution. Moreover, each block column or panel in the packed storage scheme may be considered a full ScaLAPACK matrix distributed across only one process column. This treatment of a block column panel as a particular ScaLAPACK submatrix is a key characteristic to the reuse of ScaLAPACK and PBLAS library components.

If we consider the 'local' view in process $(0,0)$, the first block column panel consists of $A_{11}, A_{31}$, $A_{51}$ and $A_{71}$. This panel is stored in memory as a $4 * m b \times n b$ Fortran column-major matrix. The second

block column panel consists of blocks $A_{54}$ and $A_{74}$. It is stored in local memory as a $2 * m b \times n b$ Fortran column-major matrix. The first entry of the second panel follows the last entry of the first panel in memory, i.e. the first entry in block $A_{54}$ follows the last entry in block $A_{71}$. Note that the entire diagonal block $A_{11}$ is stored, even though only the lower triangular part is accessed. This incurs a small price in extra storage but greatly simplifies reuse of ScaLAPACK components. 


\section{2-DIMENSIONAL BLOCK CYCLIC DISTRIBUTION}
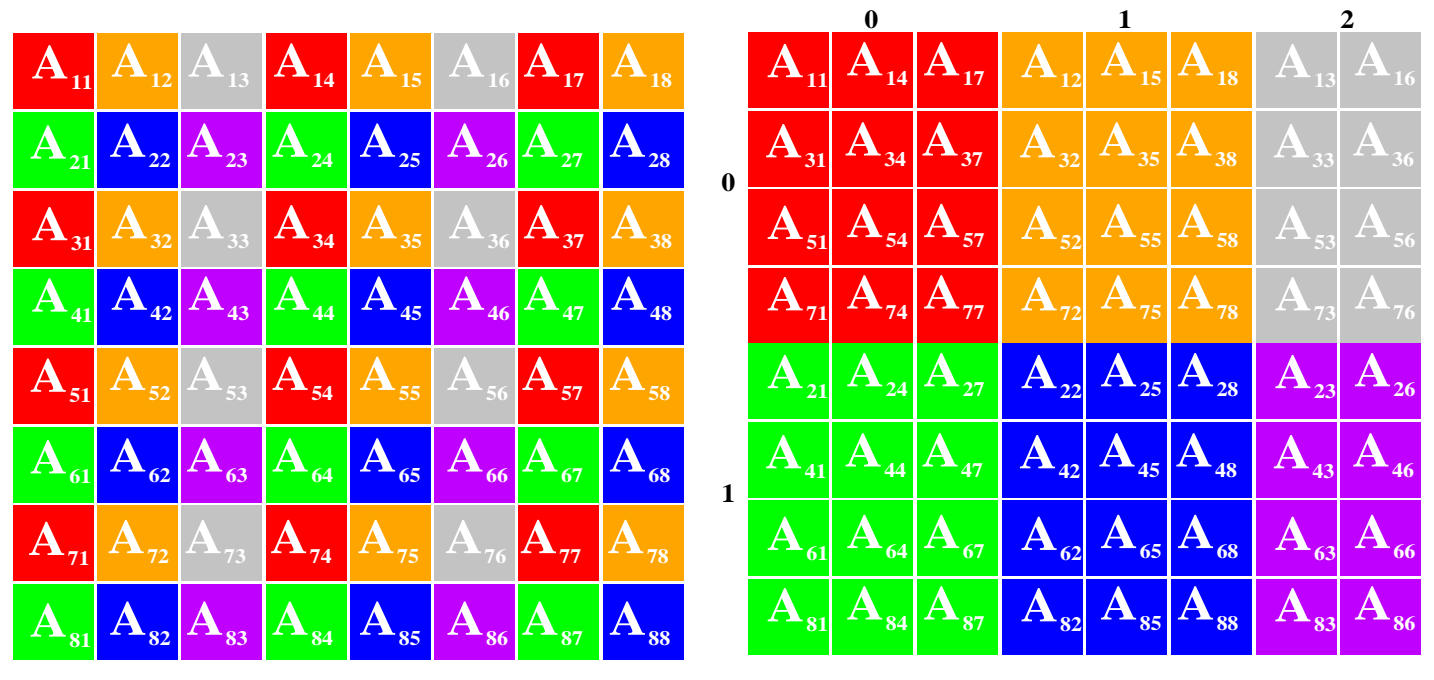

Global (left) and distributed (right) views of matrix

Figure 1: Two-dimensional block-cyclic distribution. 


\section{Examples in the use of packed storage matrix}

Here we illustrate by examples the reuse of ScaLAPACK library components for matrices stored in packed form. The key idea is the treatment of each block column or panel as a regular ScaLAPACK matrix distributed across a process column. The routine DESCINITT is provided to simplify the manipulation of indices by initializing a new matrix descriptor for a block column panel. The routine interface can be described using Fortran 90 syntax as

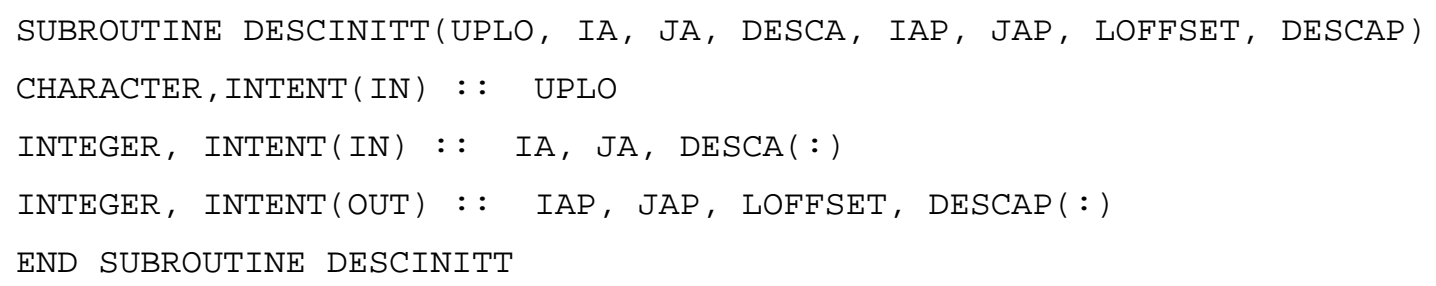

For example, access to the global entry A (IA, JA) in full storage is obtained by the ScaLAPACK routine

CALL PDELGET( SCOPE, TOP, ALPHA, A, IA, JA, DESCA)

The corresponding code to access the lower triangular entry in packed storage would be

CALL DESCINITT( 'Lower', IA, JA, DESCA, IAP, JAP, LOFFSET, DESCAP)

CALL PDELGET( SCOPE, TOP, ALPHA, A(LOFFSET), IAP, JAP, DESCAP)

The routine DESCINITT generates a new matrix descriptor DESCAP that corresponds to the block column panel with new indices (IAP, JAP) relative to the new descriptor. It will also produce the correct value for LOFFSET to adjust for the beginning of the column panel.

Another more complicated example (see Figure 2) is computing the largest absolute value $(\max (|A(I, J)|))$ in a packed matrix. This is similar to computing with the NORM= ' $\mathrm{M}$ ' option in PDLANSY for the full storage,

ANRM $=$ PDLANSY ( ' $\mathrm{M}^{\prime}, \mathrm{UPLO}, \mathrm{N}, \mathrm{A}, 1,1, \mathrm{DESCA}$, WORK$)$

The new code reuses ScaLAPACK PDLANSY and PDLANGE for computing the maximum entry in each block column panel.

The code traverses each block column (line 4) and calls DESCINITT to establish the appropriate matrix descriptor. It calls PDLANSY (line 11) to find the largest value in the diagonal block. Routine PDLANGE (line 19) computes the largest value in the remaining off-diagonal rectangular block. Although essentially the same computation is performed, the packed version has higher overhead in making several separate calls to PDLANSY and PDLANGE. Moreover, the granularity of the algorithm is limited by the width of the column panel ( $\mathrm{NB}=\mathrm{DESCA}$ (NB_) ). 


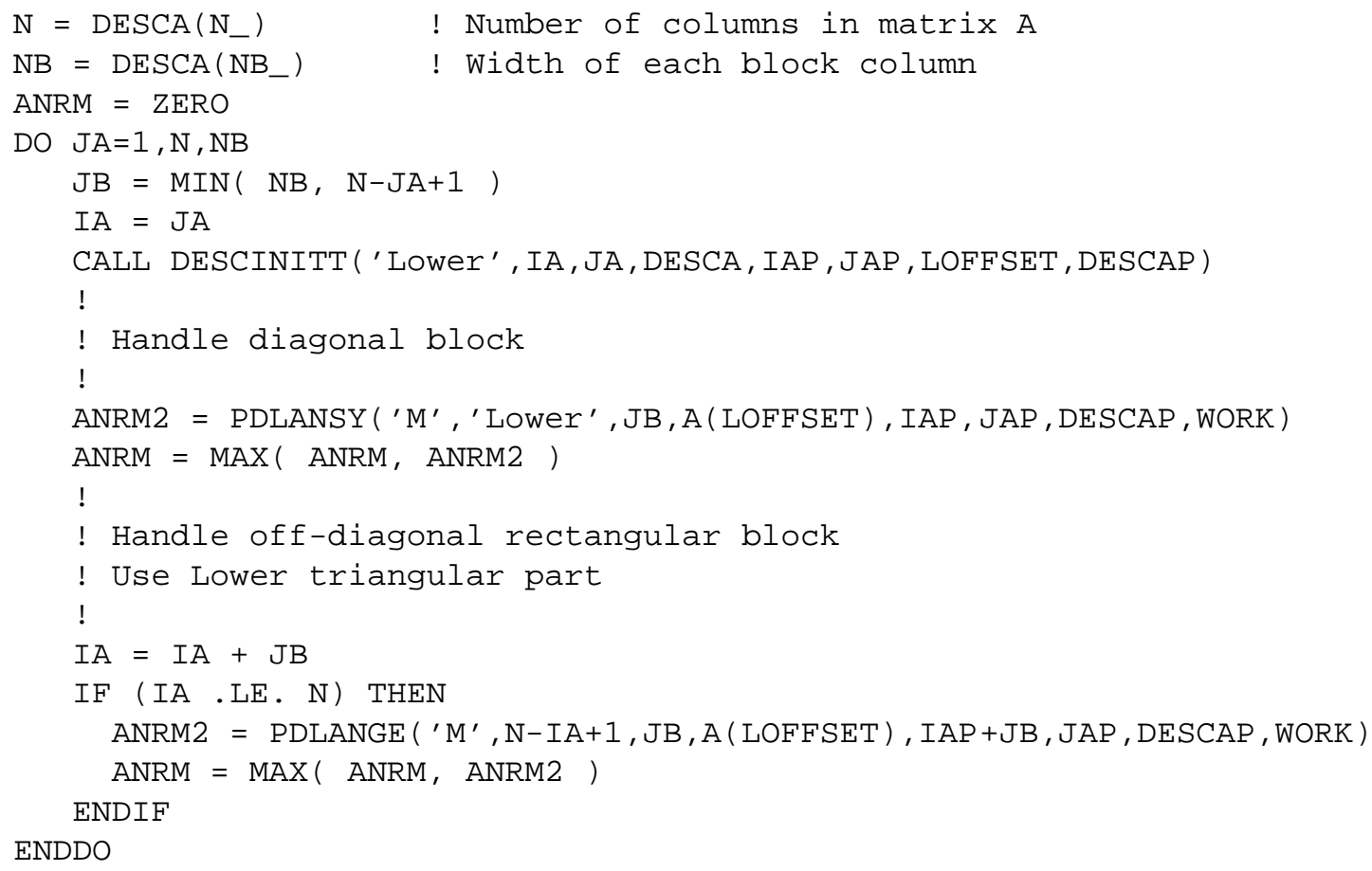

Figure 2: Example code to illustrate the reuse of ScaLAPACK components for matrices stored in packed storage. 


\section{Numerical experiments}

We have developed the following prototype codes: PxPPTRF/PxPPTRS for Cholesky factorization and solution, simple driver PXSPEV (PxHPEV) routines for finding eigenvalues and eigenvectors of symmetric (Hermitian) matrices stored in packed form, expert drivers for symmetric (Hermitian) matrices PXSPEVX/PXHPEVX and generalized eigenvalue problems PXSPGVX/PxHPGVX.

We have compared the performance of the new routines in packed storage with ScaLAPACK routines in full storage. The goal is to demonstrate that the new version with packed storage has little or no overhead cost over the existing routines for full storage. The new routines have higher overhead in index calculations and have algorithm granularity limited by the width of the block column panel. However, the packed storage may have better data locality and cache reuse.

The tests were performed on the XPS/35 Intel Paragon at the Center for Computational Sciences at the Oak Ridge National Laboratory. The XPS/35 has 512 GP nodes arranged in a $16 \times 32$ rectangular mesh. Each GP node has 32MBytes of memory. The runs were performed in a time-shared multi-user (non-dedicated) environment using a $P_{r} \times P_{c}$ logical process grid. Matrix block $m b=n b=50$ was used for all tests. Results for upper case $\left(\mathrm{UPLO}={ }^{\prime} \mathrm{U}^{\prime}\right)$ and lower case $\left(\mathrm{UPLO}={ }^{\prime} \mathrm{L}^{\prime}\right)$ are very similar so results for only the lower case are presented. The latest version of PBLAS (version 2.0 alpha) was compiled with '-O3 -Mvect -Knoieee'* and linked with '-lkmath', the highly optimized CLASSPACK serial BLAS library. The new version of PBLAS incorporates automatic algorithmic blocking with block size set to $50^{\dagger}$. The PBLAS version 2.0 alpha release is still undergoing performance tuning.

Table 1 summarizes the times for the Cholesky factorization PDPOTRF for full storage and PDPP$T R F$ for packed storage. The relative increase in runtime with packed storage over full storage is also displayed in the table. Routines PDPPTRS and PDPOTRS are used to solve the factored system with 50 and 1000 (NRHS) right-hand vectors. For the cases considered, the times for factorization by PDPPTRF with packed storage is comparable (at most two seconds difference) to times taken by PDPOTRF with full storage. Solution times for a narrow right-hand matrix (NRHS=50) show PDPPTRS for packed storage to be slower than PDPOTRSfor full storage for large problems $(N \geq 2000)$. The difference is about 3 seconds. Solution times for a wide right-hand matrix (NRHS=1000) show PDPPTRS for packed storage to be competitive with PDPPTRS. Routine PDPPTRS is slightly faster than PDPOTRS for cases $N=1000$ and $N=4000$, whereas for $N=2000$, PDPPTRS is slower by $36 \%$.

Table 2 summarizes the execution times for the symmetric eigensolvers PDSYEV with full storage and PDSPEV with packed storage. The computations were performed with $\mathrm{JOBZ}={ }^{\prime} \mathrm{N}^{\prime}$ to find all eigenvalues or with $\mathrm{JOBZ}={ }^{\prime} \mathrm{V}^{\prime}$ to find all eigenvectors and eigenvalues. Routine PDSPEV for packed storage incurs at most a $11 \%$ increase over PDSYEV for full storage in finding eigenvalues only. On

\footnotetext{
* Option -Knoieee turns off software emulation of IEEE arithmetic in divisions or operations on de-normalized numbers to use the faster (but slightly less accurate) hardware units.

$\dagger$ value return by routine P ILAENV in PBLAS.
} 
closer examination and profiling, we find part of the extra time is incurred in a routine to perform a matrix vector multiply operation where the matrix is stored in packed storage. Performance of DSYMV and DGEMV for the packed version may be limited by the width of the block column panel and by the block column by block column nature of the algorithm. When both eigenvectors and eigenvalues are required, PDSPEV compares favourably with PDSYEV for full storage.

Table 3 summarizes the execution times for the expert drivers for the symmetric eigensolvers. Although the expert driver is capable of finding specific clusters of eigenvalues, all eigenvalues (RANGE $==^{\prime} A L L^{\prime}$ ) are requested. The routine PDSPEVX performs reorthogonalization of eigenvectors when there is sufficient temporary workspace. This reorthogonalization accounts for the higher run times for finding all eigenvectors over the simple driver PDSYEV. Performance analysis of PDSYEVX is described in [2, Chapter 5] and [4]. When only eigenvalues are requested ( $\left.\mathrm{JOBZ}={ }^{\prime} \mathrm{N}^{\prime}\right)$, PDSPEVX for packed storage is slower than PDSYEVX for full storage by 5 to 9 seconds. For longer running computation when both eigenvectors and eigenvalues are requested $\left(\mathrm{JOBZ}={ }^{\prime} \mathrm{V}^{\prime}\right)$, PDSPEVX for packed storage is even slightly faster than PDSYEVX for full storage.

Table 4 summarizes the times for the generalized symmetric eigensolvers PDSPGVX with packed storage and PDSYGVX with full storage for finding all eigenvalues with RANGE $=^{\prime} \mathrm{Al} l^{\prime}$. The input parameter IBTYPE describes the type of problem to be solved:

$$
\text { Iвтүре }= \begin{cases}1 & \text { solve } A x=\lambda B x, \\ 2 & \text { solve } A B x=\lambda x, \\ 3 & \text { solve } B A x=\lambda x .\end{cases}
$$

The problem is reduced to canonical form by first performing a Cholesky factorization on $B$ ( $B=$ $L L^{H}$ or $U^{H} U$ ) and then overwriting $A$ with

$$
\text { IвTYpe }= \begin{cases}1 & A \longleftarrow U^{-H} A U^{-1} \text { or } L^{-1} A L^{-H}, \\ 2 \text { or } 3 & A \longleftarrow U A U^{H} \text { or } L^{H} A L .\end{cases}
$$

For the cases $I B T Y P E=2$ and $\operatorname{IBTYPE}=3$, the packed version incurs a significant extra overhead compared to the version for full storage. The in-place conversion of matrix $A$ to canonical form (2) may require access to block rows in matrix $A$ or $B$. Since the packed storage is stored in a column panel oriented manner, traversal across block rows will be less efficient than traversal down columns.

\section{Summary}

The overall results suggest that for a reasonably large block size $(n b=50)$, the packed storage incurs only a small time overhead over the full storage routines. The difference may be as large as $20 \mathrm{sec}-$ 


\begin{tabular}{|c|c|c|c|c|c|c|c|c|c|c|}
\hline & & & & & \multicolumn{3}{|c|}{$\mathrm{NRHS}=50$} & \multicolumn{3}{|c|}{$\mathrm{NRHS}=1000$} \\
\hline$P_{r} \times P_{c}$ & $\mathrm{~N}$ & PDPOTRF & PDPPTRF & change & PDPOTRS & PDPPTRS & change & PDPOTRS & PDPPTRS & change \\
\hline $8 \times 8$ & 1000 & $2.3 \mathrm{~s}$ & $0.9 \mathrm{~s}$ & $-63 \%$ & $1.6 \mathrm{~s}$ & $1.5 \mathrm{~s}$ & $-1 \%$ & $4.1 \mathrm{~s}$ & $3.8 \mathrm{~s}$ & $-8 \%$ \\
\hline $8 \times 8$ & 2000 & $2.5 \mathrm{~s}$ & $2.9 \mathrm{~s}$ & $14 \%$ & $1.3 \mathrm{~s}$ & $2.2 \mathrm{~s}$ & $73 \%$ & $6.9 \mathrm{~s}$ & $8.5 \mathrm{~s}$ & $24 \%$ \\
\hline $8 \times 8$ & 4000 & $14.7 \mathrm{~s}$ & $13.9 \mathrm{~s}$ & $-5 \%$ & $3.9 \mathrm{~s}$ & $7.6 \mathrm{~s}$ & $93 \%$ & $62.6 \mathrm{~s}$ & $27.8 \mathrm{~s}$ & $-56 \%$ \\
\hline $10 \times 10$ & 1000 & $2.3 \mathrm{~s}$ & $0.8 \mathrm{~s}$ & $-63 \%$ & $1.9 \mathrm{~s}$ & $1.6 \mathrm{~s}$ & $-20 \%$ & $4.2 \mathrm{~s}$ & $3.2 \mathrm{~s}$ & $-25 \%$ \\
\hline $10 \times 10$ & 2000 & $2.0 \mathrm{~s}$ & $2.5 \mathrm{~s}$ & $21 \%$ & $1.3 \mathrm{~s}$ & $2.1 \mathrm{~s}$ & $68 \%$ & $4.8 \mathrm{~s}$ & $6.5 \mathrm{~s}$ & $36 \%$ \\
\hline $10 \times 10$ & 4000 & $11.3 \mathrm{~s}$ & $10.8 \mathrm{~s}$ & $-4 \%$ & $4.1 \mathrm{~s}$ & $6.8 \mathrm{~s}$ & $67 \%$ & $58.1 \mathrm{~s}$ & $20.2 \mathrm{~s}$ & $-65 \%$ \\
\hline
\end{tabular}

Table 1: Performance (in seconds) of Cholesky factorizations and solves.

\begin{tabular}{|c|r|r||r|r|r|}
\hline$P_{r} \times P_{c}$ & \multicolumn{1}{|c|}{$\mathrm{N}$} & JOBZ & PDSYEV & PDSPEV & Change \\
\hline $8 \times 8$ & 1000 & $\mathrm{~N}$ & $25.3 \mathrm{~s}$ & $27.6 \mathrm{~s}$ & $9 \%$ \\
$8 \times 8$ & 2000 & $\mathrm{~N}$ & $81.4 \mathrm{~s}$ & $90.5 \mathrm{~s}$ & $11 \%$ \\
$8 \times 8$ & 4000 & $\mathrm{~N}$ & $317.0 \mathrm{~s}$ & $341.1 \mathrm{~s}$ & $8 \%$ \\
\hline $10 \times 10$ & 1000 & $\mathrm{~N}$ & $25.4 \mathrm{~s}$ & $27.7 \mathrm{~s}$ & $9 \%$ \\
$10 \times 10$ & 2000 & $\mathrm{~N}$ & $79.0 \mathrm{~s}$ & $87.8 \mathrm{~s}$ & $11 \%$ \\
$10 \times 10$ & 4000 & $\mathrm{~N}$ & $304.2 \mathrm{~s}$ & $321.6 \mathrm{~s}$ & $6 \%$ \\
\hline \hline $8 \times 8$ & 1000 & $\mathrm{~V}$ & $64.6 \mathrm{~s}$ & $62.8 \mathrm{~s}$ & $-3 \%$ \\
$8 \times 8$ & 2000 & $\mathrm{~V}$ & $239.6 \mathrm{~s}$ & $226.8 \mathrm{~s}$ & $-5 \%$ \\
$8 \times 8$ & 4000 & $\mathrm{~V}$ & $1336.1 \mathrm{~s}$ & $1342.3 \mathrm{~s}$ & $0 \%$ \\
\hline $10 \times 10$ & 1000 & $\mathrm{~V}$ & $65.3 \mathrm{~s}$ & $62.3 \mathrm{~s}$ & $-5 \%$ \\
$10 \times 10$ & 2000 & $\mathrm{~V}$ & $217.4 \mathrm{~s}$ & $221.7 \mathrm{~s}$ & $2 \%$ \\
$10 \times 10$ & 4000 & $\mathrm{~V}$ & $866.4 \mathrm{~s}$ & $843.2 \mathrm{~s}$ & $-3 \%$ \\
\hline
\end{tabular}

Table 2: Performance (in seconds) of simple drivers for symmetric eigensolvers.

\begin{tabular}{|c|c|r||r|r|r|}
\hline$P_{r} \times P_{c}$ & $\mathrm{~N}$ & JOBZ & PDSYEVX & PDSPEVX & Change \\
\hline $8 \times 8$ & 500 & $\mathrm{~N}$ & $6.3 \mathrm{~s}$ & $6.2 \mathrm{~s}$ & $-2 \%$ \\
$8 \times 8$ & 1000 & $\mathrm{~N}$ & $13.1 \mathrm{~s}$ & $16.0 \mathrm{~s}$ & $22 \%$ \\
$8 \times 8$ & 2000 & $\mathrm{~N}$ & $36.9 \mathrm{~s}$ & $45.4 \mathrm{~s}$ & $23 \%$ \\
\hline $10 \times 10$ & 500 & $\mathrm{~N}$ & $6.6 \mathrm{~s}$ & $6.3 \mathrm{~s}$ & $-4 \%$ \\
$10 \times 10$ & 1000 & $\mathrm{~N}$ & $11.1 \mathrm{~s}$ & $14.5 \mathrm{~s}$ & $31 \%$ \\
$10 \times 10$ & 2000 & $\mathrm{~N}$ & $34.8 \mathrm{~s}$ & $40.2 \mathrm{~s}$ & $16 \%$ \\
\hline \hline $8 \times 8$ & 500 & $\mathrm{~V}$ & $74.7 \mathrm{~s}$ & $65.9 \mathrm{~s}$ & $-12 \%$ \\
$8 \times 8$ & 1000 & $\mathrm{~V}$ & $555.1 \mathrm{~s}$ & $537.9 \mathrm{~s}$ & $-3 \%$ \\
\hline $10 \times 10$ & 500 & $\mathrm{~V}$ & $73.5 \mathrm{~s}$ & $66.0 \mathrm{~s}$ & $-10 \%$ \\
$10 \times 10$ & 1000 & $\mathrm{~V}$ & $554.4 \mathrm{~s}$ & $537.3 \mathrm{~s}$ & $-3 \%$ \\
\hline
\end{tabular}

Table 3: Performance (in seconds) of expert drivers for symmetric eigensolvers. 


\begin{tabular}{|c|c|r|r||r|r|r|}
\hline$P_{r} \times P_{c}$ & $\mathrm{~N}$ & IBTYPE & JOBZ & PDSYGVX & PDSPGVX & Change \\
\hline $8 \times 8$ & 500 & 1 & $\mathrm{~N}$ & $9 \mathrm{~s}$ & $8 \mathrm{~s}$ & $-11 \%$ \\
$8 \times 8$ & 1000 & 1 & $\mathrm{~N}$ & $15 \mathrm{~s}$ & $21 \mathrm{~s}$ & $34 \%$ \\
$8 \times 8$ & 2000 & 1 & $\mathrm{~N}$ & $54 \mathrm{~s}$ & $70 \mathrm{~s}$ & $30 \%$ \\
\hline $8 \times 8$ & 500 & 2 & $\mathrm{~N}$ & $6 \mathrm{~s}$ & $7 \mathrm{~s}$ & $15 \%$ \\
$8 \times 8$ & 1000 & 2 & $\mathrm{~N}$ & $15 \mathrm{~s}$ & $21 \mathrm{~s}$ & $38 \%$ \\
$8 \times 8$ & 2000 & 2 & $\mathrm{~N}$ & $48 \mathrm{~s}$ & $75 \mathrm{~s}$ & $55 \%$ \\
\hline $8 \times 8$ & 500 & 3 & $\mathrm{~N}$ & $5 \mathrm{~s}$ & $7 \mathrm{~s}$ & $31 \%$ \\
$8 \times 8$ & 1000 & 3 & $\mathrm{~N}$ & $15 \mathrm{~s}$ & $21 \mathrm{~s}$ & $39 \%$ \\
$8 \times 8$ & 2000 & 3 & $\mathrm{~N}$ & $48 \mathrm{~s}$ & $75 \mathrm{~s}$ & $55 \%$ \\
\hline $10 \times 10$ & 500 & 1 & $\mathrm{~N}$ & $10 \mathrm{~s}$ & $8 \mathrm{~s}$ & $-16 \%$ \\
$10 \times 10$ & 1000 & 1 & $\mathrm{~N}$ & $14 \mathrm{~s}$ & $20 \mathrm{~s}$ & $39 \%$ \\
$10 \times 10$ & 2000 & 1 & $\mathrm{~N}$ & $47 \mathrm{~s}$ & $62 \mathrm{~s}$ & $32 \%$ \\
\hline $10 \times 10$ & 500 & 2 & $\mathrm{~N}$ & $6 \mathrm{~s}$ & $7 \mathrm{~s}$ & $14 \%$ \\
$10 \times 10$ & 1000 & 2 & $\mathrm{~N}$ & $13 \mathrm{~s}$ & $19 \mathrm{~s}$ & $41 \%$ \\
$10 \times 10$ & 2000 & 2 & $\mathrm{~N}$ & $40 \mathrm{~s}$ & $65 \mathrm{~s}$ & $62 \%$ \\
\hline $10 \times 10$ & 500 & 3 & $\mathrm{~N}$ & $5 \mathrm{~s}$ & $7 \mathrm{~s}$ & $36 \%$ \\
$10 \times 10$ & 1000 & 3 & $\mathrm{~N}$ & $13 \mathrm{~s}$ & $19 \mathrm{~s}$ & $44 \%$ \\
$10 \times 10$ & 2000 & 3 & $\mathrm{~N}$ & $40 \mathrm{~s}$ & $65 \mathrm{~s}$ & $61 \%$ \\
\hline \hline $8 \times 8$ & 500 & 1 & $\mathrm{~V}$ & $12 \mathrm{~s}$ & $10 \mathrm{~s}$ & $-19 \%$ \\
$8 \times 8$ & 1000 & 1 & $\mathrm{~V}$ & $21 \mathrm{~s}$ & $25 \mathrm{~s}$ & $18 \%$ \\
$8 \times 8$ & 2000 & 1 & $\mathrm{~V}$ & $76 \mathrm{~s}$ & $89 \mathrm{~s}$ & $16 \%$ \\
\hline $8 \times 8$ & 500 & 2 & $\mathrm{~V}$ & $7 \mathrm{~s}$ & $8 \mathrm{~s}$ & $25 \%$ \\
$8 \times 8$ & 1000 & 2 & $\mathrm{~V}$ & $19 \mathrm{~s}$ & $25 \mathrm{~s}$ & $33 \%$ \\
$8 \times 8$ & 2000 & 2 & $\mathrm{~V}$ & $66 \mathrm{~s}$ & $94 \mathrm{~s}$ & $42 \%$ \\
\hline $8 \times 8$ & 500 & 3 & $\mathrm{~V}$ & $6 \mathrm{~s}$ & $8 \mathrm{~s}$ & $29 \%$ \\
$8 \times 8$ & 1000 & 3 & $\mathrm{~V}$ & $18 \mathrm{~s}$ & $25 \mathrm{~s}$ & $34 \%$ \\
$8 \times 8$ & 2000 & 3 & $\mathrm{~V}$ & $65 \mathrm{~s}$ & $93 \mathrm{~s}$ & $44 \%$ \\
\hline $10 \times 10$ & 500 & 1 & $\mathrm{~V}$ & $13 \mathrm{~s}$ & $10 \mathrm{~s}$ & $-25 \%$ \\
$10 \times 10$ & 1000 & 1 & $\mathrm{~V}$ & $18 \mathrm{~s}$ & $23 \mathrm{~s}$ & $27 \%$ \\
$10 \times 10$ & 2000 & 1 & $\mathrm{~V}$ & $64 \mathrm{~s}$ & $76 \mathrm{~s}$ & $19 \%$ \\
\hline $10 \times 10$ & 500 & 2 & $\mathrm{~V}$ & $6 \mathrm{~s}$ & $8 \mathrm{~s}$ & $28 \%$ \\
$10 \times 10$ & 1000 & 2 & $\mathrm{~V}$ & $16 \mathrm{~s}$ & $22 \mathrm{~s}$ & $38 \%$ \\
$10 \times 10$ & 2000 & 2 & $\mathrm{~V}$ & $53 \mathrm{~s}$ & $79 \mathrm{~s}$ & $49 \%$ \\
\hline $10 \times 10$ & 500 & 3 & $\mathrm{~V}$ & $6 \mathrm{~s}$ & $8 \mathrm{~s}$ & $34 \%$ \\
$10 \times 10$ & 1000 & 3 & $\mathrm{~V}$ & $16 \mathrm{~s}$ & $22 \mathrm{~s}$ & $39 \%$ \\
$10 \times 10$ & 2000 & 3 & $\mathrm{~V}$ & $52 \mathrm{~s}$ & $78 \mathrm{~s}$ & $51 \%$ \\
\hline
\end{tabular}

Table 4: Performance (in seconds) of expert drivers for generalized eigensolvers. 
onds for short runs that complete in about a minute and approximately extra $10 \%$ overhead for larger problems. In some cases, the packed storage may even yield slightly better performance due to better data locality and cache reuse. The generalized eigensolver with $\triangle B T Y P E=2$ or $\triangle B T Y P E=3$ may require traversal across block rows and this leads to higher overhead (about 30\% - 50\%) for packed storage over full storage.

The design of the packed storage data layout to be a dense ScaLAPACK matrix in each block column panel also facilitates the reuse of PBLAS and ScaLAPACK library components for good performance. 


\section{References}

[1] E. Anderson, Z. Bai, C. Bischof, J. Demmel, J. Dongarra, J. D. Croz, A. Greenbaum, S. Hammarling, A. McKenney, S. Ostrouchov, And D. SORENSEN, LAPACK Users' Guide, SIAM, second ed., 1995. Online version at http://www. netlib.org/lapack/lug/lapack_lug.html.

[2] L. S. Blackford, J. Choi, A. Cleary, E. D’Azevedo, J. Demmel, I. Dhilon, J. Dongarra, S. Hammarling, G. Henry, A. Petitet, K. Stanley, D. WalkER, AND R. C. Whaley, ScaLAPACK Users' Guide, SIAM, 1997. Online version at http://www. netlib.org/scalapack/slug/scalapack_slug.html.

[3] J. Choi, J. Dongarra, S. Ostrouchov, A. Petitet, D. Walker, And R. C. Whaley, A proposal for a set of parallel basic linear algebra subprograms, Tech. Rep. CS-95-292, Department of Computer Science, University of Tennessee, Knoxville, Tennessee, 1995. Also appears as LAPACK working note 100. Online version at http://www. netlib.org/lapack/lawns/lawn100.ps.

[4] J. Demmel And K. Stanley, The performance of finding eigenvalues and eigenvectors of dense symmetric matrices on distributed memory computers, Tech. Rep. CS-94-254, Department of Computer Science, University of Tennessee, Knoxville, Tennessee, 1994. Also appears as LAPACK working note 86. Online version at http://www. netlib.org/lapack/lawns/lawn86.ps. 